\title{
Control of Dual Mode Power Split Transmission for a Hybrid Electric Vehicle
}

\author{
Namdoo Kim*, Jeongmin Kim*, and Hyunsoo Kim*
}

Motor control algorithm for a dual power split system is proposed for hybrid electric vehicles (HEV). The dual mode power split system consists of an electric variator, MG1 and MG2, and three planetary gear sets. In order to develop the control algorithm for the best fuel economy, the dual mode PST is analyzed by network analysis. From the network analysis results, it is found that the dual mode PST efficiency decreases in the speed ratios where the power circulation occurs, and it is required that the ICE needs to be operated on the speed ratio region where the powertrain efficiency is relatively high. Using the dynamic models of the HEV powertrain, a motor control algorithm to obtain the high system efficiency is designed by inversion-based control. In order to evaluate performance of the control algorithm, HEV simulator is developed using Cruise and MATLAB Simulink. It is found from the simulation results that the motor control algorithm proposed in this study provides improved fuel economy since the motor control is able to provide the ICE operation on the speed ratio range, which gives relatively high powertrain system efficiency.

Keywords: PST (Power Split Transmission), HEV (Hybrid Electric Vehicle), Dual Mode

\section{INTRODUCTION}

Growing concerns on saving energy and preventing global warming have been pressing the automotive industry to produce automobiles with less exhaust emission and better fuel economy. Under these circumstances, a hybrid electric vehicle (HEV) is considered to be a viable solution to meet those requirements in short to mid-term. Various hybrid architectures have been proposed and implemented over the past ten years. Series hybrid is conceptually the simplest hybrid. However, poor efficiency and large electric machine size limits its applicability to specific vehicle types. Parallel hybrids, which use one or more electric motors, have been used in many passenger car applications since the electric machines can be sized only for the desired functions rather than the full engine power and drivetrain efficiency is relatively high [1].

Besides the series and parallel hybrids, a power-split type can be added to series and parallel hybrids. In the power-split transmission, the input power can be split into two parts, one of which is delivered to the wheels via the variator, which controls the speed ratio, and the other via a purely mechanical path which consists of planetary gear sets [2]. This principle is known from hydro-mechanical transmissions (HMTs). In HMTs, hydraulic pump and motor set is used as the variator,

*Sungkyunkwan University

300 Chunchun-dong, Suwon`si, Gyeonggi-do, 440-746, Korea and provides an infinite speed ratio [3]. The power-split concept has been recently extended to include electric power paths rather than hydraulic path to create electro-mechanical, power-split infinitely variable transmissions, known as PST in short [4]. The Toyota Prius is the most prominent representative of PSTs of this kind [5].

Since the PST is capable of providing an infinite input-to-output speed ratio range, including a geared neutral, the engine can be directly connected to the transmission at all times independent of the vehicle speed. A major advantage of this PST architecture stands in the possibility to de-couple the internal combustion engine (ICE) and wheel speed as long as the output power demand is met; this gives much more flexibility to choose the ICE working point in order to optimize fuel consumption and reduce exhaust emission [6]. But the PST is characterized by internal power circulation. In the PST, the internal power circulation occurs along the closed loop depending on the speed ratio, and sometimes the circulated power increases enormously, which requires a variator size that is $2 \sim 3$ times larger than the input power. This power circulation can lead to high losses, and thereby to a low efficiency of the power transmission. It is noted that the power circulation is the primary reason why the THS (Toyota hybrid system) shows relatively low efficiency in the high speed region.

In order to avoid or minimize the power circulation of 
the PST recently, a dual mode PST which is named as 'the dual mode' in contrast to 'the single mode' like the THS, has been investigated as a design alternative for the HEV transmission to achieve the improved system efficiency in overall operating speed ranges. Various dual mode PST design configurations have been proposed as seen in recent patents and publications [7 10].

In design of the dual mode PST hybrid electric vehicle, a control strategy considering the overall powertrain efficiency should be required. In general, the PST system efficiency varies depending on the speed ratio due to its power transmission characteristics. An existing HEV control strategy which operates the IC engine on the best thermal efficiency region may deteriorate the overall system efficiency. In addition, since the dual mode PST is operated by two motor-generators, control algorithm of each motor is required by considering dynamics of the PST, IC engine and the vehicle. In this paper, a control algorithm on how to operate the IC engine and motors is proposed for a dual mode PST from the viewpoint of the overall powertrain system efficiency. First, power characteristic of the dual mode PST used in this study is investigated by network analysis and the dual mode PST system efficiency is obtained with respect to the speed ratio. Based on the network analysis results, a motor torque control algorithm is proposed, which provides the motor speed to operate the IC engine for the best powertrain efficiency.

\section{CHARACTERISTICS OF DUAL MODE PST}

\subsection{Network Theory}

In Figure 1, a dual mode PST investigated in this study is shown. The dual mode PST in Figure 1 consists of three planetary gears, two clutches and an electric variator which is composed of two motors: MG1 and MG2. The IC engine is connected to the ring gear R1 of the planetary gear PG1 and the motor MG1 is connected to the sun gear S1 of PG1 and the sun gear S2 of the planetary gear PG2. The dual mode PST in Figure 1 realizes the mode 1 by engaging the brake $\mathrm{B}$ (Figure $2 \mathrm{a}$ ) and mode 2 by engaging the clutch CL (Figure $2 \mathrm{~b}$ ).

Power characteristic of the dual mode PST in Figure 1 is analyzed by network theory. Network theory by Hedman [11,12] can be summarized as follows: a general mechanical power transmission system consists of power transmission elements (TM element) and shafts. TM elements are connected to shafts and are represented by nodes. Speed and torque equations can be obtained at each node.

Speed equation at node: Speed at node $\mathrm{j}$ can be determined from relationship between neighbor speed nodes.

$$
\vec{M}_{\omega} \cdot \vec{\omega}=\overrightarrow{\beta_{\omega}}
$$

where $M_{\omega}$ is the square matrix consisting of the coefficient of speed relationship, $\omega$ is the speed vector as $\omega^{T}=\left[\begin{array}{cccc}\omega_{1} & \omega_{2} & \ldots & \omega_{n}\end{array}\right]$, and $\beta_{\omega}$ is the boundary vector consisting of known input (or output) speeds.

Torque equation at node: When $\mathrm{k}$ torque nodes are connected at jth speed node, the following torque equation can be obtained from torque equilibrium of the jth shaft.

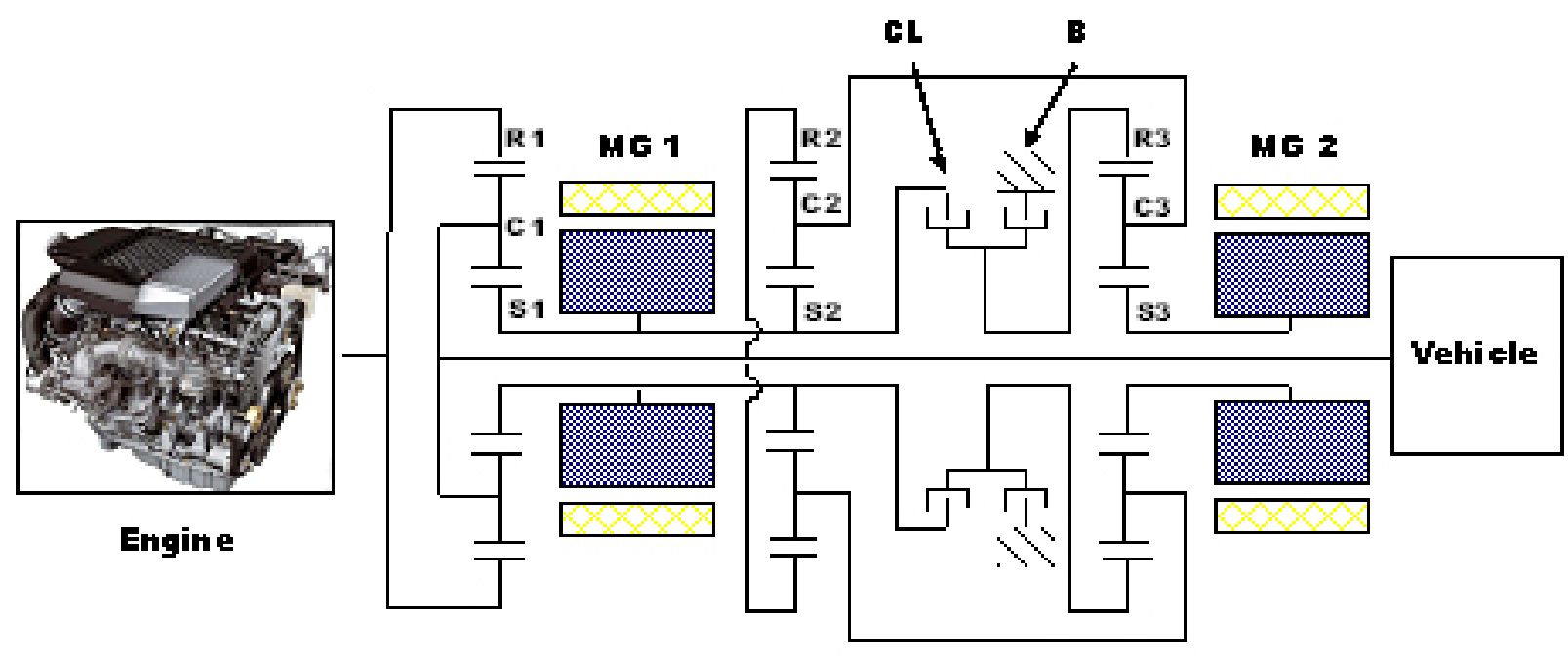

PG 1 PG 2 PG

Figure 1: Schematic diagram of dual mode PST system 


$$
\sum_{i=j}^{j+k} T_{i}=0 \quad \text { for } j=1,2, \ldots, N_{n}
$$

where $i$ is the torque node number at jth speed node. For TM elements, torque equation can be obtained as

$$
\vec{M}_{T} \cdot \vec{T}=\overrightarrow{\beta_{T}}
$$

where $M_{T}$ is the square matrix which consists of the coefficients of torque equations, $T$ is the torque vector as $T_{T}=\left[T_{1} T_{2} \ldots T_{N S}\right]$, and $\beta_{T}$ is the boundary vector.

\subsection{Network Analysis of Dual Mode PST}

In Figure 2, network model of the dual mode PST is shown. In the network analysis, it is assumed that the power loss is only attributed to the torque loss. The power variator model is shown in Figure 2 as TM element. The dual mode PST has two operation modes, depending on clutch and brake operation. For mode 1, the brake B is applied, so the speed of the ring gear 3 is zero (Figure 2a). The torque and speed matrices for mode 1 in Figure 2 can be obtained as (below)

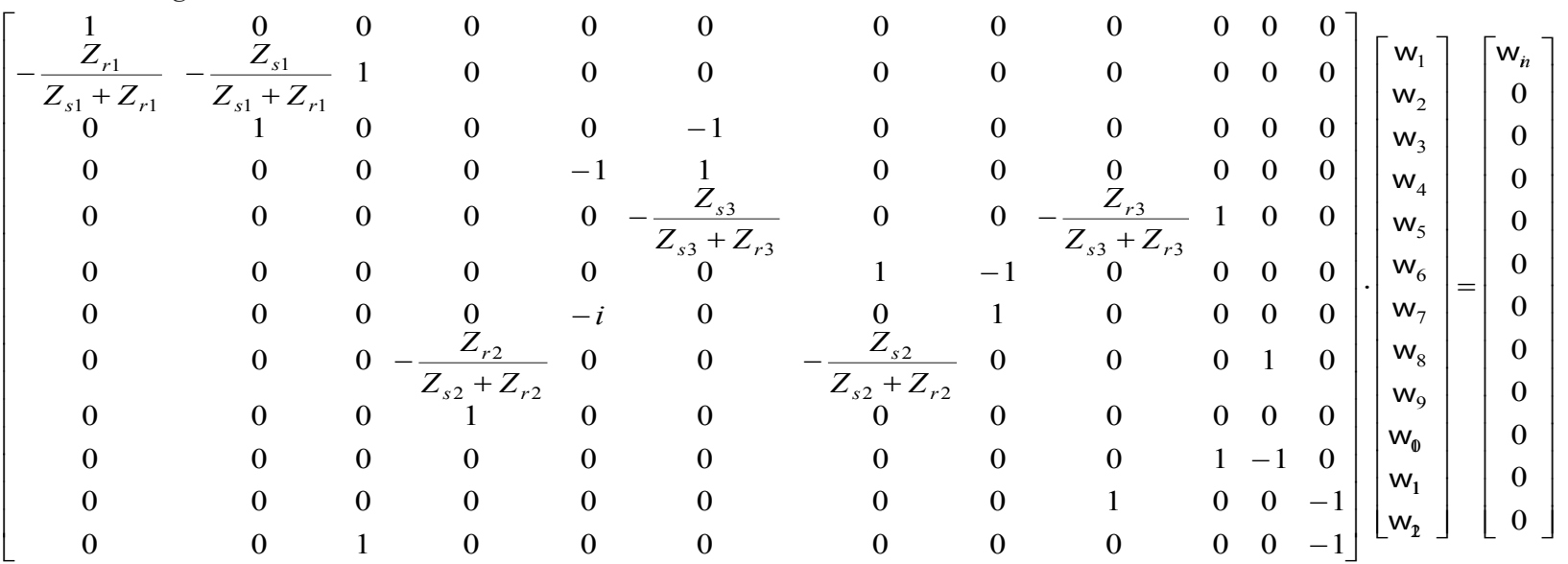

$$
\begin{aligned}
& {\left[\begin{array}{cccccccccccc}
1 & 0 & 0 & 0 & 0 & 0 & 0 & 0 & 0 & 0 & 0 & 0 \\
1 & 1 & 1 & 0 & 0 & 0 & 0 & 0 & 0 & 0 & 0 & 0 \\
-Z_{s 1} & Z_{r 1} & 0 & 0 & 0 & 0 & 0 & 0 & 0 & 0 & 0 & 0 \\
0 & 1 & 0 & 0 & 1 & 1 & 0 & 0 & 0 & 0 & 0 & 0 \\
0 & 0 & 0 & 1 & 0 & 0 & 1 & 0 & 0 & 0 & 1 & 0 \\
0 & 0 & 0 & -Z_{s 3} & 0 & 0 & Z_{r 3} & 0 & 0 & 0 & 0 & 0 \\
0 & 0 & 0 & 0 & 0 & 0 & 1 & 1 & 0 & 0 & 0 & 0 \\
0 & 0 & 0 & 0 & 1 & 0 & 0 & i & 0 & 0 & 0 & 0 \\
0 & 0 & 0 & 0 & 0 & 1 & 0 & 0 & 1 & 1 & 0 & 0 \\
0 & 0 & 0 & 0 & 0 & Z_{r 2} & 0 & 0 & -Z_{s 2} & 0 & 0 & 0 \\
0 & 0 & 0 & 0 & 0 & 0 & 0 & 0 & 0 & 1 & 1 & 0 \\
0 & 0 & 1 & 0 & 0 & 0 & 0 & 0 & 1 & 0 & 0 & 1
\end{array}\right] \cdot\left[\begin{array}{c}
T_{1} \\
T_{2} \\
T_{3} \\
T_{4} \\
T_{5} \\
T_{6} \\
T_{7} \\
T_{8} \\
T_{9} \\
T_{0} \\
T_{1} \\
T_{1}
\end{array}\right]=\left[\begin{array}{c}
T_{i} \\
0 \\
0 \\
0 \\
0 \\
0 \\
0 \\
0 \\
0 \\
0 \\
0 \\
0
\end{array}\right]}
\end{aligned}
$$




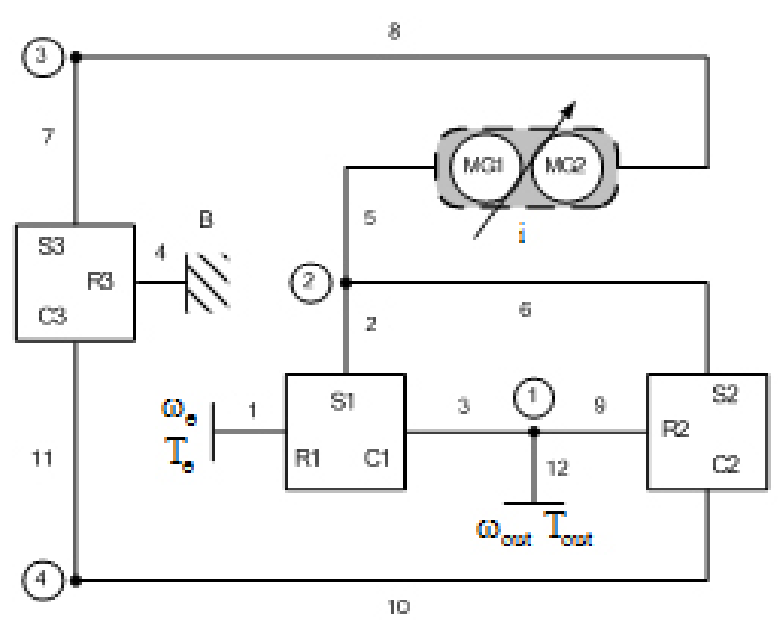

(a) Mode 1

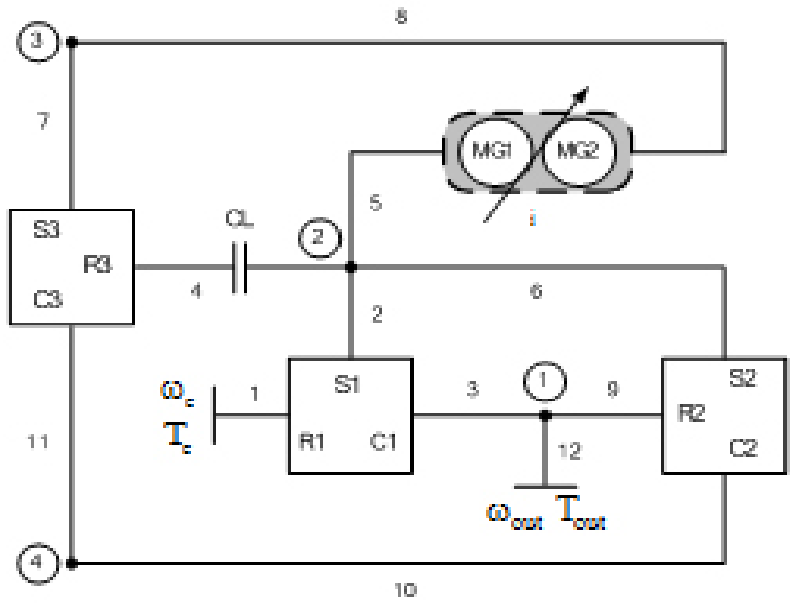

(b) Mode 2

Figure 2: Network model for dual mode PST system

ratio reaches $\mathrm{SR}=0.75$. At $\mathrm{SR}=0.75$, the dual mode $\mathrm{PST}$ shows the highest efficiency because all the power is transmitted only through mechanical part. At this point, the shift from mode 1 to mode 2 is carried out by the clutch and brake operation. The system efficiency at mode 2 is relatively high because the power ratio of the electric part at mode 2 is smaller than the power ratio of the electric part at mode 1 until speed ratio becomes $\mathrm{SR}=1.2$.

In Figure 4 , negative value of $P_{M G 1} / P_{\varepsilon}$ means that the power direction through the electric variatior is revered; in other words, power circulation occurs along the closed loop. Once the power circulation occurs, the PST efficiency decreases due to the relatively low efficiency of the electric variatior. For instance, at $\mathrm{SR}=0.2,75 \%$ of the engine input power passes through the electric variatior and the PST efficiency shows $69 \%$ (Figure 5a).

If $\mathrm{SR}$ is somewhere between $\mathrm{A}$ and $\mathrm{B}$ or between $\mathrm{B}$ and $\mathrm{C}$, the input power is divided into the electric part and the mechanical part, and no power circulation occurs. As shown in Figure 5b, when SR=0.6, 15\% of the input power passes through the electric variator and the remaining power goes to the mechanical part. For $\mathrm{B}<\mathrm{SR}<\mathrm{C}$, even if $P_{M G 1} / P_{\varepsilon}$ shows a negative value, it is expected that the PST efficiency is high since the magnitude of the circulation is small (Figure 5a and Figure 5c). When $\mathrm{SR}$ is larger than point $\mathrm{C}$ in mode $2, P_{M G 1} / P_{\varepsilon}$ shows a positive value. If $P_{M G 1} / P_{\varepsilon}$ is larger than 1, it also means that power circulation occurs. For instance, at $\mathrm{SR}=1.85$, the electric power ratio is 1.35 , which means that $135 \%$ of the input power passes through the variator.

From Figure 4, it is found that the PST system should

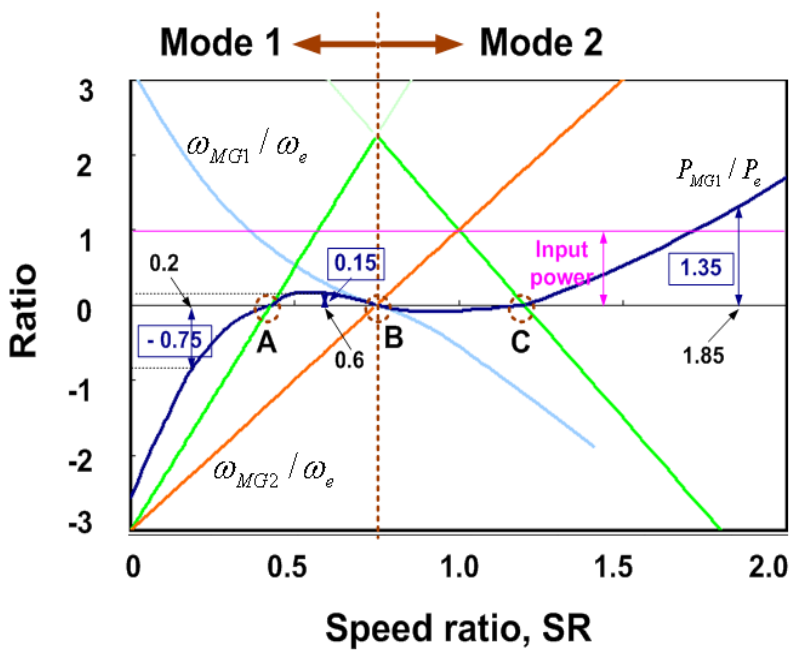

Figure 3: Network analysis results for dual mode PST

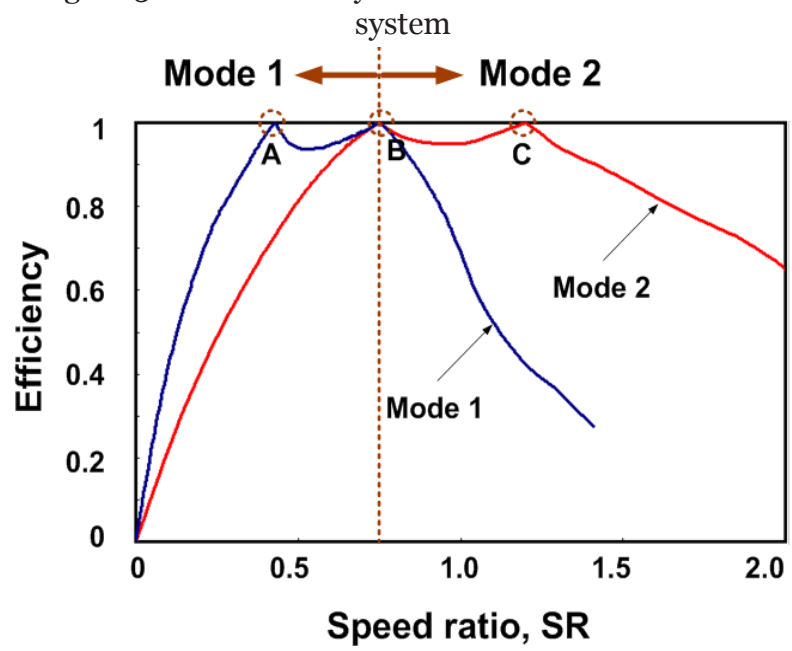

Figure 4: Network analysis results for dual mode PST system 


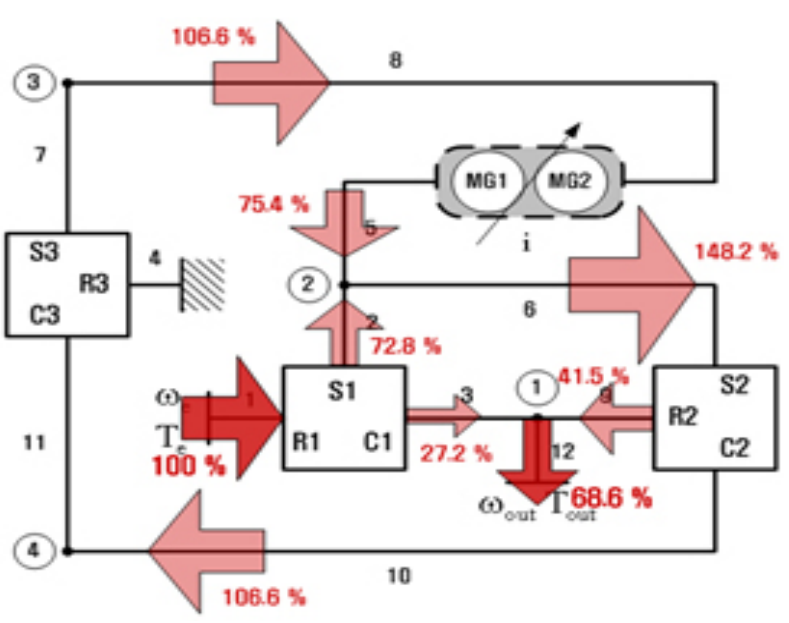

(a) $\mathrm{SR}=0.2$

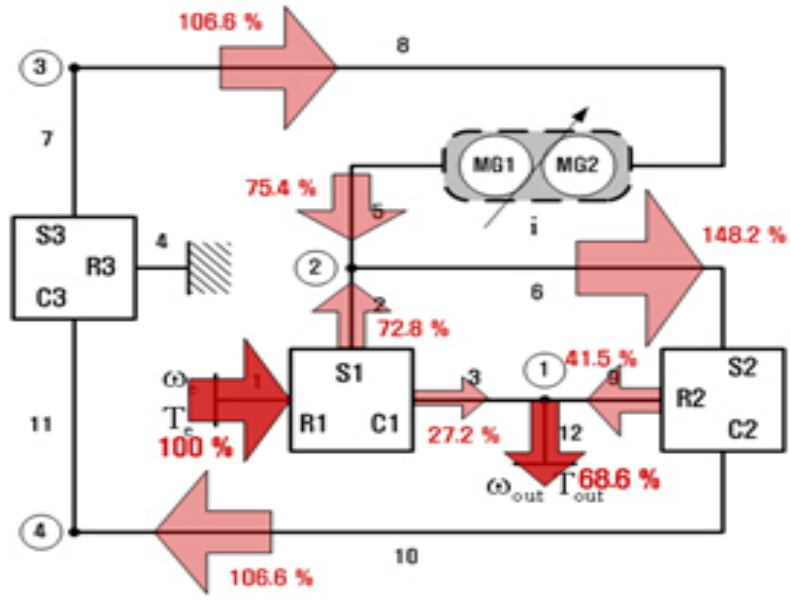

(b) $\mathrm{SR}=0.6$

Figure 5: Power flow of PST

be operated on the speed ratios where the PST efficiency is relatively high. In the conventional CVT vehicle, the CVT ratio is controlled only from the viewpoint of the engine thermal efficiency. In other words, the CVT ratio control is performed to maintain the engine operation on the optimal operating region which is selected to obtain the minimum fuel consumption. However, in the PST vehicle, it is noted from Figure $3 \sim$ Figure 5 the engine operation speed needs to be that selected to maintain the speed ratio SR which provides the high PST efficiency. Since the vehicle velocity is determined by the driver's demand, the engine speed can be controlled by the motors MG1 and MG2.

\section{DUAL MODE PST CONTROL}

\subsection{Dynamic Modeling of HEV Powertrain}

In order to design a control algorithm for the MG1 and MG2 to obtain the high powertrain efficiency, dynamic model of the dual mode PST needs to be constructed.

For the powertrain modeling, the following assumptions are made:

o Inertia of the pinion gears is neglected because it is relatively small and there is no interest in the torque acting on the pinion gears,

o All shafts within the powertrain are assumed to be rigid,

o Sun gear inertia is lumped with MG1 rotor inertia,

o Inertia of the ring gear is lumped with MG2 rotor inertia,

o Carrier inertia is lumped with engine inertia.

From the assumptions, a bond graph representing the powertrain can be constructed, as shown in Figure 6 .
From the bond graph, dynamic equations of the dual mode PST powertrain can be easily obtained. Note that only two differential equations are required to represent the powertrain system since there are only two independent state variables - engine speed ( $\omega e)$ and vehicle velocity $(V)$. With simple mathematical manipulation, the dynamic equations can be obtained as follows

$$
\alpha \cdot \dot{X}=\beta \cdot u
$$

where $X=\left[\omega_{\varepsilon} V\right]^{T}, \mathrm{u}=\left[\mathrm{T}_{\varepsilon} T_{M G 1} T_{M G 2} F_{L}\right]^{T}$

$$
\begin{aligned}
\alpha= & {\left[\begin{array}{c}
J_{e}+\frac{b^{2} J_{M G 1}}{a^{2}}+\frac{(b d f-b)^{2} J_{M G 2}}{a^{2} d^{2} e^{2}} \\
-\frac{b N_{d} J_{M G 1}}{a^{2} R_{t}}+\frac{(b d f-b \gamma 1+a c-d f) N_{d} J_{M G 2}}{a^{2} d^{2} e^{2} R_{t}} \\
\quad \frac{b N_{d} J_{M G 1}}{a^{2} R_{t}}+\frac{(b d f-b \gamma 1+a c-d f) N_{d} J_{M G 2}}{a^{2} d^{2} e^{2} R_{t}} \\
M+\frac{N_{d}^{2} J_{M G 2}}{a^{2} R_{t}^{2}}+\frac{(1+a c-d f)^{2} N_{d}^{2} J_{M G 2}}{a^{2} d^{2} e^{2} R_{t}^{e}}+\frac{2 J_{w}}{R_{t}^{2}}
\end{array}\right] }
\end{aligned}
$$

where $J$ is the lumped inertia of engine and carrier gear, $J_{M G 1}, J_{M G 2}$ is the motor inertia, $J_{w}$ is the wheel inertia, $N_{d}$ is the gear ratio from the ring gear to driveshaft, $R_{t}$ is the tire radius, $M$ is the vehicle mass and $F_{L}$ is the road load (e.g. aerodynamic drag, rolling resistance, and road grade). From Equation (6), dynamics of the dual mode power split HEV powertrain can be represented in state-space equation as follows:

$$
\begin{aligned}
& \dot{X}=A \cdot X+B \cdot u \\
& Y=C \cdot X+D \cdot u
\end{aligned}
$$




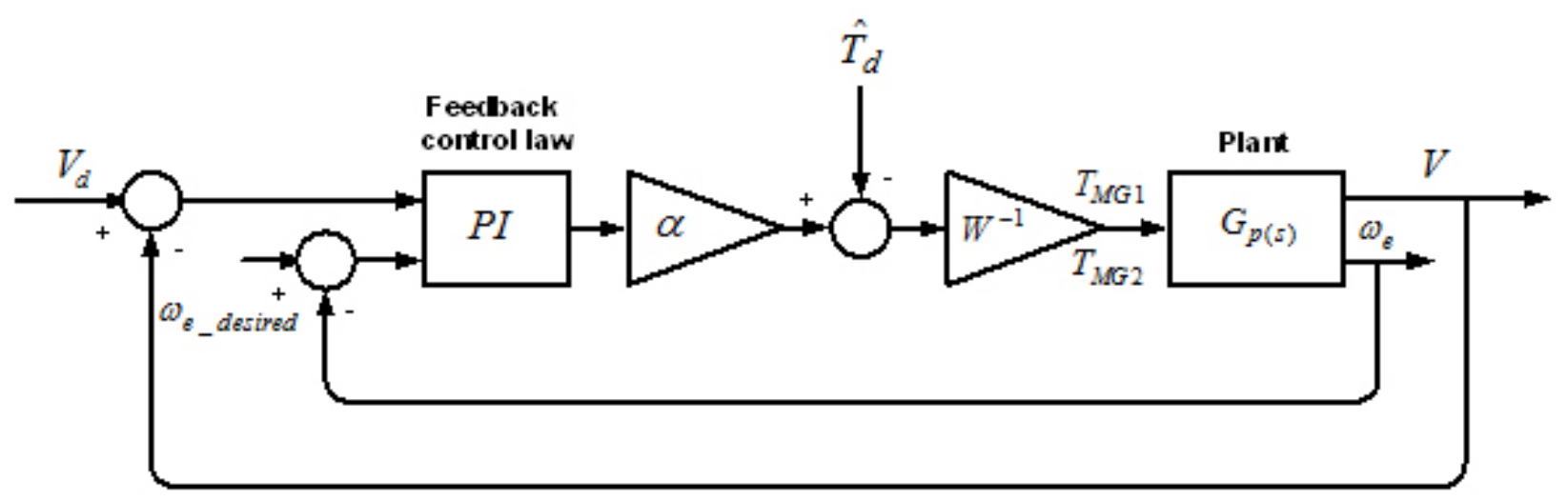

Figure 6: Control diagram

where $\left.Y=\omega_{\iota} \omega_{M G 1} \omega_{M G 2} V\right]^{T}, \quad A=\left[\begin{array}{ll}0 & 0 \\ 0 & 0\end{array}\right]$, and $B=\alpha^{-1} \bullet \beta, \quad T_{d}=\left[\begin{array}{c}T_{e} \\ F_{L}\end{array}\right]$

$C=\left[\begin{array}{cc}1 & 0 \\ -\frac{b}{a} & -\frac{N_{d}}{a R} \\ \frac{(b d f-b)}{a d e} & \frac{(1+a c-d f) N_{d}}{a d e R_{t}} \\ 0 & 1\end{array}\right] D=\left[\begin{array}{llll}0 & 0 & 0 & 0 \\ 0 & 0 & 0 & 0 \\ 0 & 0 & 0 & 0 \\ 0 & 0 & 0 & 0\end{array}\right]$

where $a, b, c, d, e$, and $f$ are the planetary gear ratio.

\subsection{Design of Control System}

It is seen from Equation (6) (7) that the dual mode PST powertrain has two state variables: the engine speed $\omega_{e}$ and vehicle speed $V$, and four control inputs: the engine torque $T_{e}$, MG1 torque $T_{M G 1}, \mathrm{MG} 2$ torque $T_{M G 2}$ and road load $F_{L}$. However, among four control inputs, only $\mathrm{T}_{\mathrm{MG} 1}$ and $\mathrm{T}_{\mathrm{MG} 2}$ can be used to control the state variables $\omega_{e}$ and $\mathrm{V}$ since the engine torque $T_{e}$ depends on the driver's accelerator pedal operation to follow the driving schedule and road load $\mathrm{F}_{\mathrm{L}}$ is determined by the vehicle running condition, such as air drag and road grade.

In order to find out $\mathrm{T}_{\mathrm{MG} 1}$ and $\mathrm{T}_{\mathrm{MG} 2}$ that provide the desired engine speed for higher system efficiency, Equation (6) is reconstructed by introducing the matrix $\mathrm{W}$

$$
\beta \cdot u=W \cdot u_{w}+T_{d}
$$

where $W=\left[\begin{array}{cc}-\frac{b}{a} & -\frac{(b-b d f)}{a d e} \\ \frac{N_{d}}{a R_{t}} & \frac{(a c+1-d f) N_{d}}{a d e R_{t}}\end{array}\right], u_{w}=\left[\begin{array}{c}T_{M G 1} \\ T_{M G 2}\end{array}\right]^{(8)}$,
From Equation (7) and Equation (8), state equation can be obtained as

$$
\dot{X}=\alpha^{-1} \cdot W \cdot u_{w}+\alpha^{-1} \cdot T_{d}
$$

Assuming that the differences between the actual value $\alpha, W, T_{D}$ and the calculated value are less than $\varepsilon_{i}$ that is small enough, the control input $u_{w}$ can be defined as

$$
\begin{aligned}
u_{w} & =\hat{W}^{-1} \hat{\alpha}\left(-K_{P}\left[X-X_{d}\right]-K_{I} \int_{0}^{t}\left[X-X_{d}\right]+\dot{X}_{d}-\hat{W}^{-1} \hat{T}_{d}\right. \\
& =W^{-1} \alpha\left(-K_{P}\left[X-X_{d}\right]-K_{I} \int_{0}^{t}\left[X-X_{d}\right]+\dot{X}_{d}\right)-W^{-1} T_{d}+\widetilde{u}_{1}-\widetilde{u}_{2}
\end{aligned}
$$

where $X_{d}$ is the desired state, $K_{p}$ is the proportional gain, and $K_{I}$ is the integral gain. $\tilde{u}_{1}, \tilde{u}_{2}$ can be obtained

$$
\begin{gathered}
\tilde{u}_{1}=\left(\hat{W}^{-1} \hat{\alpha}-W_{\alpha}^{-1} \gamma-K_{P}\left[X-X_{d}\right]-K_{I} \int_{0}^{t}\left[X-X_{d}\right]+\dot{X}_{d}\right) \\
\tilde{u}_{2}=\left(\hat{W}^{-1} \hat{T}_{d}-W^{-1} T_{d}\right)
\end{gathered}
$$

Now we can solve Equation (11) by selecting proper control gain $K_{P}$ and $K_{I}$, which gives the error $\ell$ which converges into zero fast enough and the control input $u_{w}$; in other words, $\mathrm{T}_{\mathrm{MG} 1}$ and $\mathrm{T}_{\mathrm{MG} 2}$ can be obtained from Equation (10) as follows: 
In Figure 6, a control block diagram for dual mode PST is shown. For the desired vehicle velocity $V_{d}$, the desired engine speed $\omega_{\varepsilon \text {-desired }}$ can be determined, which maintains the dual mode PST operation in relatively high efficiency range; in other words, to maintain the speed ratio between $0.43<\mathrm{SR}<1.2$ (Figure 3 ). The speed ratio control can be achieved by the motor torque $\mathrm{T}_{\mathrm{MG} 1}$ and $\mathrm{T}_{\mathrm{MG} 2}$ from Equation (12).

\subsection{HEV Control Strategy}

In order to evaluate the dual mode PST control algorithm, a hybrid electric vehicle control strategy is required at first. In this study, it is assumed that the PST HEV is operated by the following threeoperation modes.

EV mode: the vehicle is propelled only by the electric motors. The vehicle runs in EV mode under the following conditions:

o the demanded vehicle power is less than the electric motor maximum power

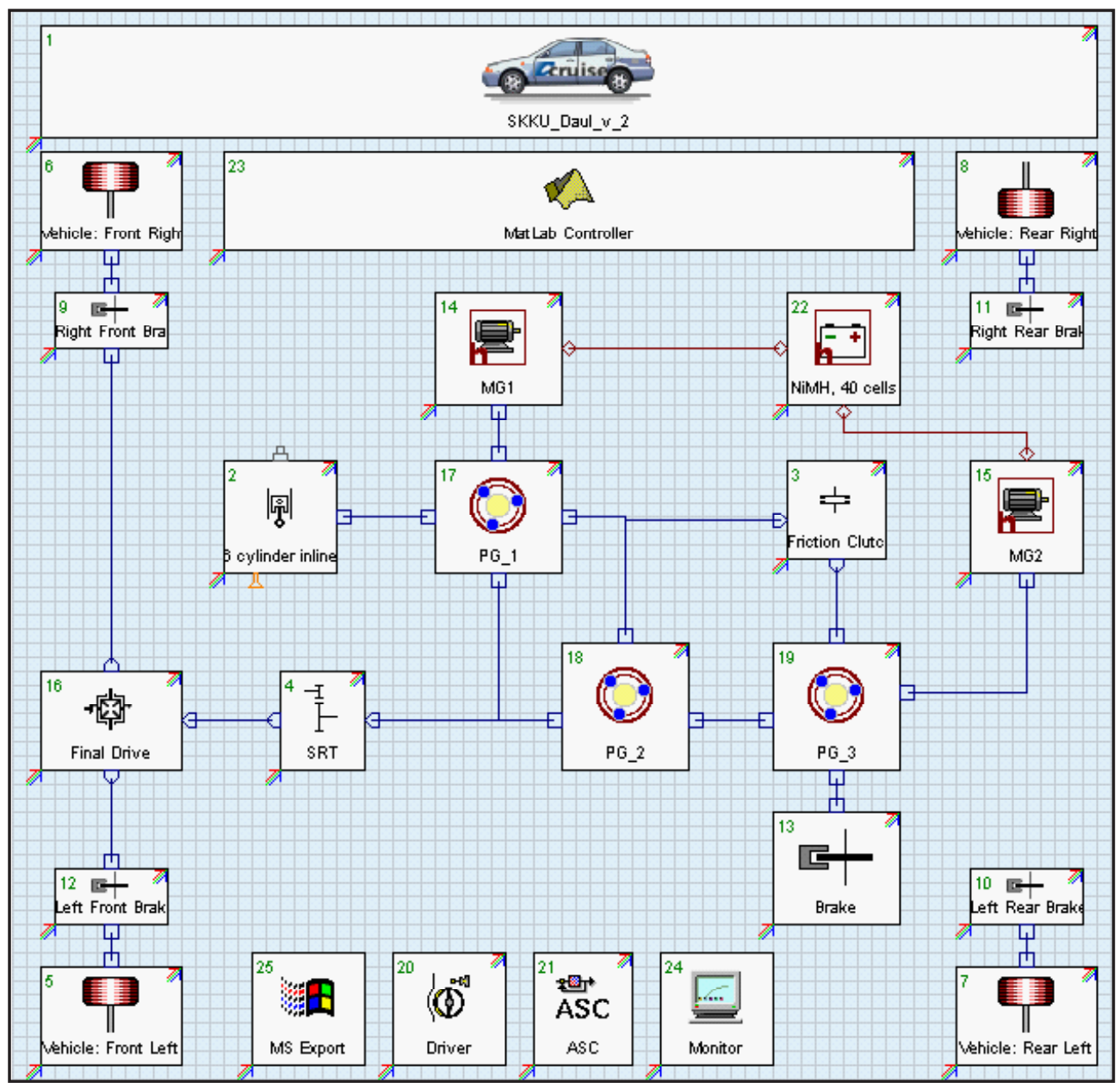

$o$ the battery state of charge (SOC) is higher than the lower limit

o the vehicle velocity is less than the threshold velocity (generally the vehicle is operated in EV mode at start)

HEV mode: If the driver's accelerator pedal opening is large enough or the battery SOC drops below the lower limit, the vehicle is propelled by the engine and the motor, and if necessary, the battery is charged by the engine. In the HEV mode, the PST control algorithm proposed in this study is used for the higher system efficiency.

Deceleration mode: In deceleration mode, regenerative braking is carried out. The required electric motor torque is calculated from the demanded braking force by considering the electric motor efficiency.

\section{SIMULATION RESULTS AND DISCUSSION}

In order to evaluate performance of the motor control algorithm proposed in this study, a hybrid electric vehicle simulator is developed. Dynamic models of the HEV powertrains are obtained with Cruise software and control algorithm is modeled using MATLAB Simulink. In Figure 7, Cruise simulator models such as ICE, PST, electric motor, battery, and tire are shown.

Using the simulator, performance of the motor control algorithm is investigated. In the simulation, fuel economy of the HEV is evaluated using (1) the control algorithm from the viewpoint of

Figure 7: Cruise model for dual mode PST system 

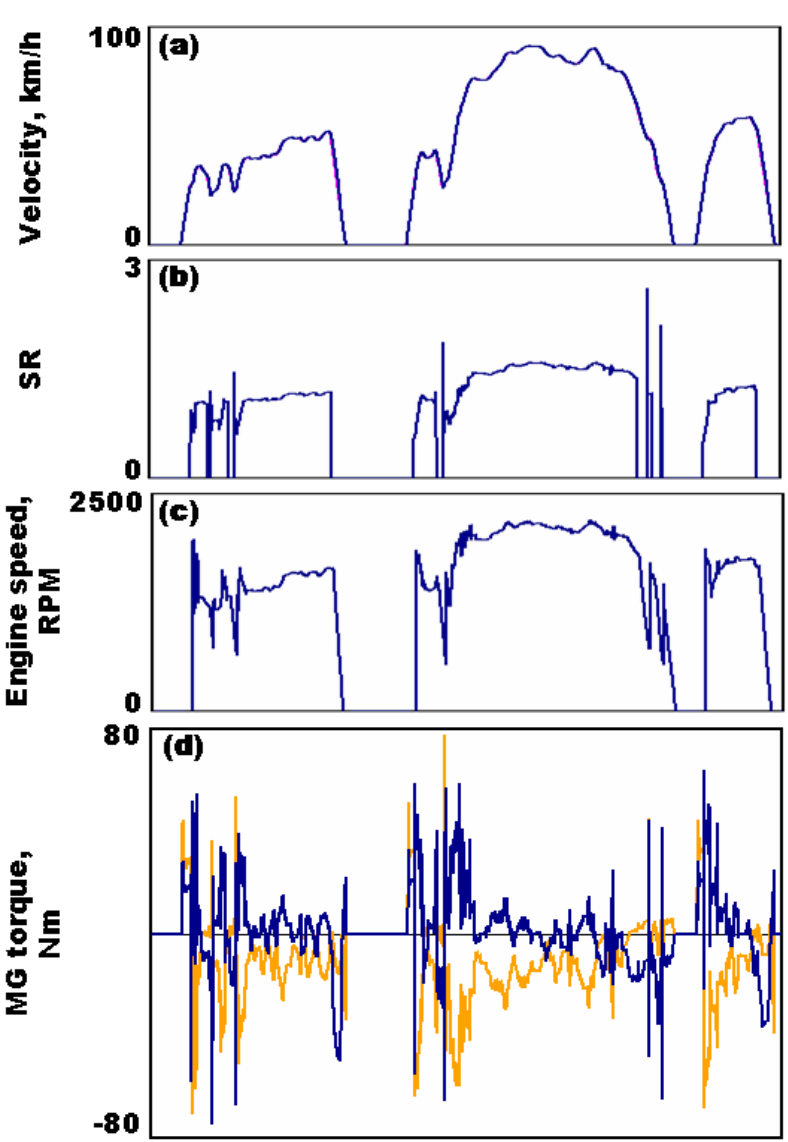

Time, sec

Figure 8: PTE control

the whole powertrain efficiency (PTE control) and (2) the optimal operating line (OOL) for the ICE (OOL control). For the PTE control, the ICE speed $\omega_{e}$ is controlled by the MG1 and MG2 to provide the speed ratio SR, which gives higher powertrain system efficiency in Figure 4. For the OOL control, the MG1 and MG2 are controlled to operate the ICE on the OOL for the minimum fuel consumption from the viewpoint of the engine thermal efficiency.

In Figure $8 \sim 9$, simulation results are compared for the first $0 \sim 400$ seconds of FUDS (federal urban driving schedule). The vehicle velocity follows the target velocity closely for the two control algorithms. However, the speed ratio SR shows different value for each control algorithm. In the PTE control (Figure 8), $\mathrm{SR}$ remains around $\mathrm{SR}=1.4$ between two node points ( $\mathrm{SR}=0.43 \sim 1.2)$ for most of the driving schedule where the powertrain efficiency is relatively high (Figure 4); meanwhile SR for the OOL control (Figure 9) changes from $\mathrm{SR}=1.1$ to $\mathrm{SR}=2.7$. The ICE for the PTE control (Figure 8c) is operated on relatively high speed region
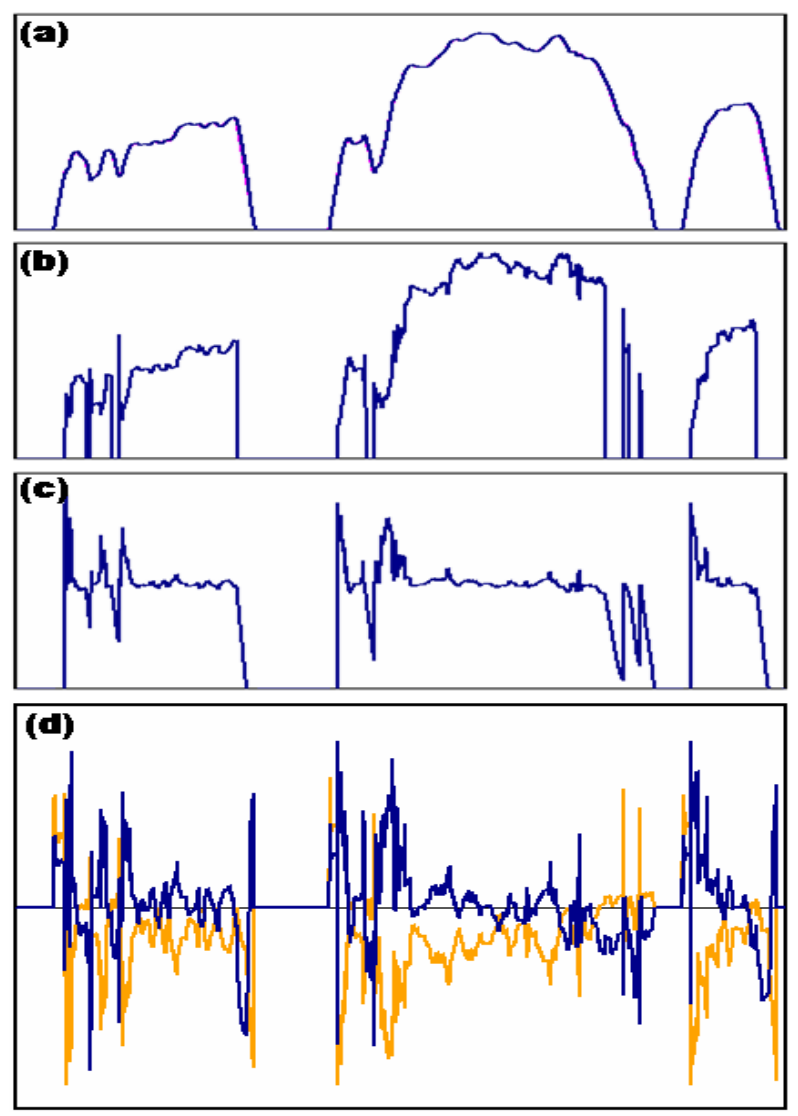

Time, sec

Figure 9: OOL control

compared with that of the OOL control (Figure 9c). It is seen from Figure $9 \mathrm{c}$ that the ICE is operated mostly on the optimal operating speed around $1200 \mathrm{rpm}$ by the OOL control. The motor torque shows positive value in the EV mode. And the MG1 is used as a motor and the MG2 is used as a generator for control algorithm in HEV mode.

In Figure 10, the ICE operation trajectory is compared for two control algorithms. It is noted that the engine operation is carried out in relatively high speed and low torque region for the PTE control (Figure 10a), compared with those of the OOL control (Figure 10b). Even if the engine operation is performed out of the OOL for the PTE control, the fuel economy of the PTE control is improved by $4.8 \%$ (Figure 11) since the PST is operated on the speed ratio range where the powertrain efficiency is relatively high.

\section{CONCLUSION}

Motor control algorithm for a dual power split system 

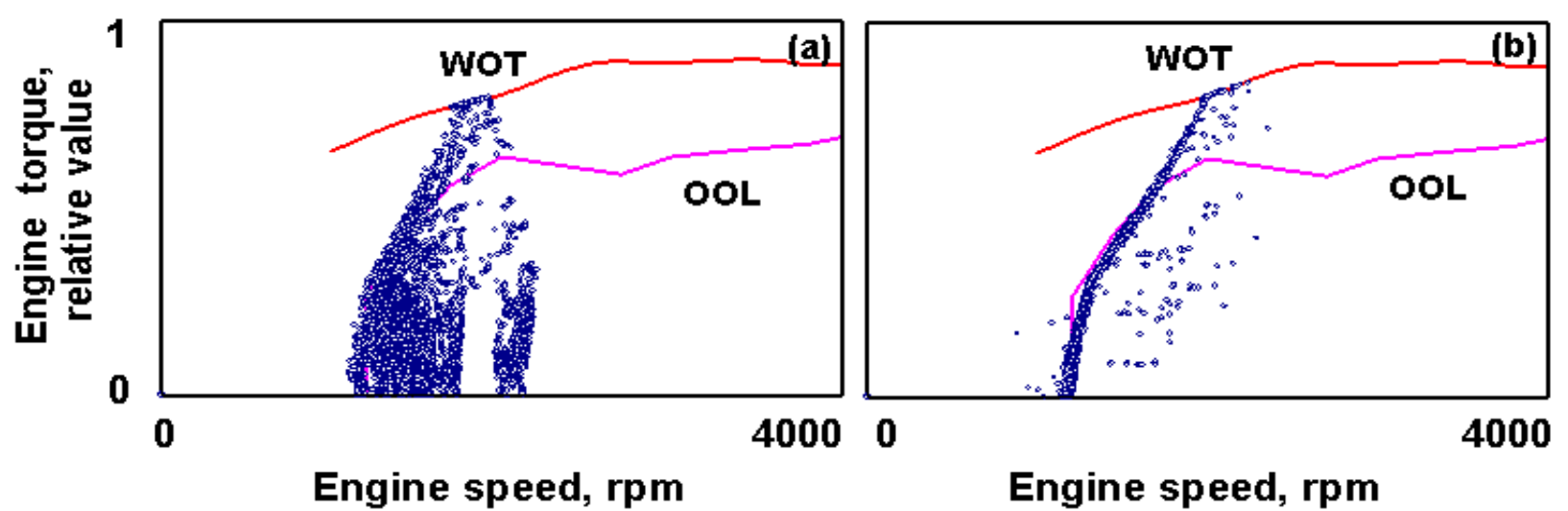

Figure 10:Comparison of engine operation trajectory for (a) PTE control and (b) OOL control

is proposed for hybrid electric vehicles (HEV). The dual mode power split system consists of an electric variator, MG1 and MG2, and three planetary gear sets. In order to develop the control algorithm for the best fuel economy, the dual mode PST is analyzed by network analysis. From the network analysis results, it is found that the dual mode PST efficiency decreases in the speed ratios where the power circulation occurs, and it is required that the ICE needs to be operated on the speed ratio region where the powertrain efficiency is relatively high.

Using the dynamic models of the HEV powertrain, a motor control algorithm to obtain the high system efficiency is designed by inversion-based control. In order to evaluate performance of the control algorithm, HEV simulator is developed using Cruise and MATLAB Simulink. It is found from the simulation results that the motor control algorithm proposed in this study provides improved fuel economy since the motor control is able to provide the ICE operation on the speed ratio range, which gives relatively high powertrain system efficiency.

\section{REFERENCES}

[1] Conlon, B. Comparative Analysis of Single and Combined Hybrid Electrically Variable Transmission Operating Modes. SAE Paper 2005-01-1162, 2005.

[2] Yaegashi, T., Sasaki, S. and Abe, T. Toyota Hybrid System : It's Concept and Technologies. Proceedings of FISTA98, Paris, 1998, F98TP095.

[3] Sung, D. H., Hwang, S. H. and Kim, H. S. Design of Hydromechanical Transmission using Network Analysis. Journal of Automobile Engineering, 2005, 219, 53-63.

[4] Schmidt, M. R. Two-Mode, Split Power, Electromechanical Transmission. US Patent, 1996, 5577973.

[5] Muta, K., Yamazaki, M. and Tokieda, J. Development

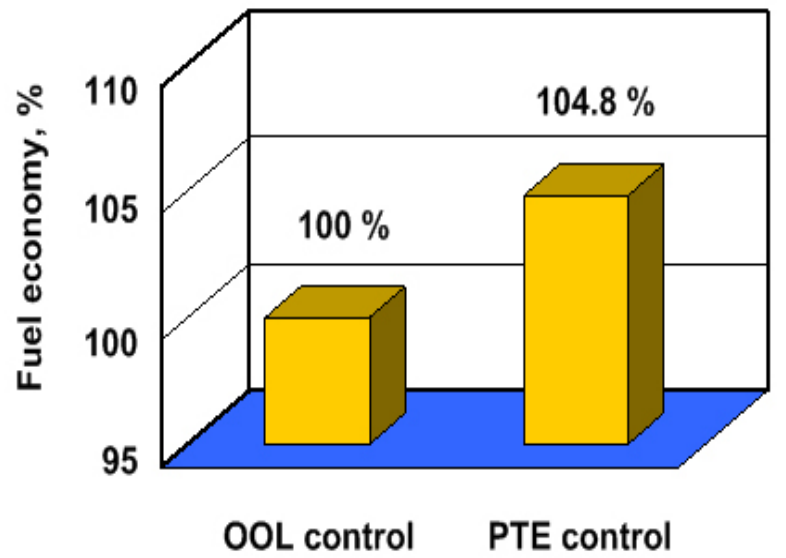

Figure 11: Comparison of fuel economy

of New-Generation Hybrid System THS II - Drastic Improvement of Power Performance and Fuel Economy. SAE Paper 2004-01-0064, 2004.

[6] Ai, X., Mohr, T. and Anderson, S. An ElectroMechanical Infinitely Variable Speed Transmission for Hybrid Electric Vehicle. SAE Paper, 2005-01-0281, 2005.

[7] Velleneuve, A. Dual Mode Electric Infinitely Variable Transmission. Proceedings of International Congress on Continuously Variable Power Transmission. CVT '04, San Francisco, 04CVT-19, 2004.

[8] Imazu, T. and Kargar, K. Hybrid Transmission Control System. US Patent, 2004, 20040149501.

[9] Holmes, A. and Schmidt, M. Three-mode, Compound-split, Electrically-variable Transmission. US Patent, 2003, 20030078126.

[10] Ai, X. and Mohr, T. Continuously Variable Transmission. US Patent, 2003, 6595884.

[11] Hedman, A. A Method to Analyze mechanical transmission systems. Report no. 1985-11-08, pp.9 13, 
Division of Machine Elements, Chalmers University of Technology, Sweden, 1985.

[12] Hedman, A. Computer-aided analysis of general mechanical transmission system - some examples. 2nd Int. Conference on New Development in Power Train and Chassis Engineering, Strassburg, France, 1989.

\section{AUTHORS}

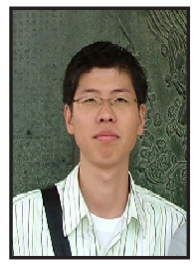

Namdoo Kim, Graduate Student, School of Mechanical Engineering, Sungkyunkwan University, 300 Chunchun-dong, Suwon, Gyeonggi-do, 440-746, Korea

Phone : 82-31-290-7473

Fax : 82-31-290-7679

wever8@skku.edu

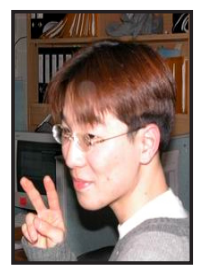

Jeongmin Kim, PhD Student, School of Mechanical Engineering, Sungkyunkwan University, 300 Chunchun-dong, Suwon, Gyeonggi-do, 440-746, Korea

Phone : 82-31-290-7473

Fax : 82-31-290-7679

pcpppp@empal.com

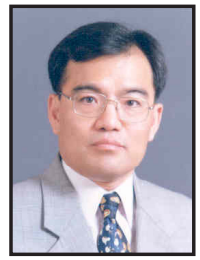

Lead Author Hyunsoo Kim, Professor, School of Mechanical Engineering, Sungkyunkwan University, 300 Chunchun-dong, Suwon-si, Gyeonggido, 440-746, Korea

Phone : 82-31-290-7438

Fax : 82-31-290-7679

hskim@me.skku.ac.kr 